\title{
Re-ranking with Click-Base Similarity and Typicality using Spectral Clustering
}

\author{
Mayuri Kawalkar ${ }^{1}$, Gangotri Nathaney ${ }^{2}$ \\ M. Tech scholar, CSE Department, WCEM College, Nagpur, India ${ }^{1}$ \\ Assistant Professor, CSE Department, WCEM College, Nagpur, India ${ }^{2}$
}

\begin{abstract}
Application on World Wide Web and internet is increasing exponentially. A huge amount of database is added every minute so there is need for effective and efficient image searching. In order to improve image search performance image re-ranking system are proposed, but now days there some problem arises like semantic gap, intent gap which restrict development in image retrieval. This paper present a novel re-ranking approach name spectral clustering re-ranking with clicked base similarity and typicality. We use image click through data as implicit feedback from user and help to overcome intention gap. Proposed system contains saliencing technique. Saliency region contain more important information in the image. So less time require for searching. Our re-ranking approach can significantly improve search results, and outperform several existing re-ranking approaches.
\end{abstract}

Keywords: spectral clustering, image search, clicked through data, typicality.

\section{INTRODUCTION}

Hundreds of thousands of images are uploaded to the internet with the explosive growth of online social media and the attractiveness of capture devices thus, building satisfying image retrieval system is the key to improve user search experience. In order to improve search performance, image search re-ranking, which adjusts the initial ranking orders by mining visual content or leveraging some auxiliary knowledge is proposed, and has been the focus of attention in both academia and industry in recent years. Most of the existing re- ranking system use visual information and give limited performance. They neglect "intent gap" ( the gap between representation of user query and the real intent of the user). The user real intent is very hard to capture without user ${ }^{\mathrm{s}}$ participation and feedback. Therefore the search engine record the queries issued by user and corresponding clicked images. Although the clicked images along with their quires cannot reflect explicit user preferences. We develop a novel image search re-ranking approach, named spectral clustering re-ranking with click-based similarity and typicality (SCCST). In image search re-ranking named spectral clustering re-ranking with clicked based similarity and typicality which use first use image click information to guide image similarity learning for multiple features, and then carry out spectral clustering to group visually and semantically similar images into clusters. Finally achieve the re-ranking results by calculating click-based clusters typicality and within-clusters click based image typicality in descending order. In clicked base multi feature similarity learning uses click through data and multiple modalities simultaneously to learn image similarity. It increases the image search performance. To improve image categorization result proposed Re-Ranking based image searching using saliencing technique. Re-ranking provide proper image classification. Saliency detection techniques can be used to approximate the foreground and background regions according to the saliency distribution. During the diffusion process, the image gradients in the salient regions are increased while those in non-salient regions are decreased. The background information gradually fades out while the foreground information is preserved and important structures in the foreground are enhanced. The saliency driven multi-scale space of an image can be used to handle unsure background information. After saliency driven nonlinear diffusion, an image is represented by the set of its multi-scale images and the fusion of information from different scales. Then apply re-ranking. Saliency driven nonlinear multi-scale image representation has several advantages. In the nonlinear scale space, semantically important image structures are preserved or improved at large scales, and the locations of the important image structures are not shifted after diffusion at any scale. The section II describes the related work. Section III describes the proposed technique in briefly. Section IV describe the experimental result and conclusion in section V.

\section{RELATED WORK}

XiaopengYang,TaoMei,Jie Liu proposed novel re-ranking spectral clustering re-ranking with click base similarity and typicality. It use clicked through data to direct image similarity for click multiple feature and then conduct spectral clustering and obtained re-ranking calculating clicked base cluster typicality. For these feature extraction technique like edge,color feature extraction technique are used. 
Xiaopeng Yang, yangdongzhang, Ting Yao, Tao Mei proposed new re-ranking algorithm name click boosting multimodality graph base re-ranking. The algorithm leverages click image to put similar image that are not clicked. And reranked them in multimodality graph base scheme. The clicked through data can be viewed as footprint of search user behaviour. The feature extraction technique like color moment autocorologram, gabber wavelet, texture wavelet are use for the feature extraction to compare the feature of similar image.

JunieCai, Zheng,Junzha, Mengwang, prposedattribute assisted re-ranking. In these each image is represented by an attribute feature consisting the response from the classifier. A hyper graph is used to model the relationship between images by integrating low level feature and semantic feature. For visually similar images the feature are extracted. The edge distribution algorithm used for material attribute. $\mathrm{K}$ means is use for clustering. The svm classifier used for classification images.

Yongdong Zhang, Xiapong Yang, Tao Mei proposed re-ranking algorithm called clicked base relevance feedback. it levegres clicked images as positive data and images from query as negative data to improve classification accuracy and automatically learn the fusion weight of each modality. The svm classifier used for the classification of images.

Yan Liu, Tao Mei, Xian Sheng, Xiuqing Wu proposed novel approach to visual search re-ranking.

\section{PROPSED METHODOLOGY}

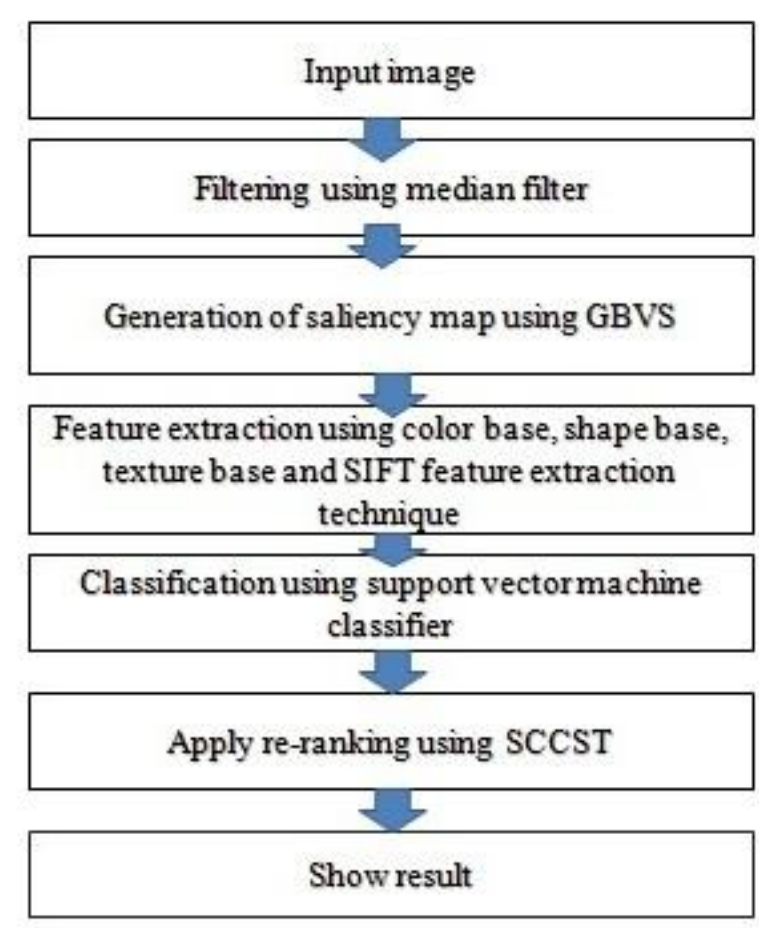

Fig 3.1: proposed methodology

The above diagram shows step in proposed system. The first step is image is given as input instead of text querry. Then pre-processing technique used. The next step is use graph base visual saliency. Then the feature extraction technique is used. Then classification using SVM classifier and then applies spectral clustering re-ranking with clicked base similarity and typicality. All technique isdescribed briefly in below.

- $\quad$ Image pre-processing:

The pre-processing technique is use to remove distortion or noise. We use median filter for the pre-processing $>$ Medain filter:

It removes all type of noise. It averages the intensity of all neighbours pixel.It provides efficient noise decrease capability with considerable less blurring than any other filter. Hence we use median filter.

- $\quad$ Saliency map generation using GBVS:

Saliency should be defined as the discriminativeness of features. Saliency maps have information about where interesting information can be initiate in the image. These areas correspond to features considered as singular or informative, depending on the definition of saliency. High saliency regions correspond to objects or places they are most likely to be found, while lower saliency is associated to background. Graph-Based Visual Saliency (GBVS) is 
consists of two steps: first forming activation maps on certain feature channels, and then normalizing them in a method which highlights conspicuity and admits combination with other maps.

Graph Based Visual Saliency (GBVS) steps:

1. Compute raw feature map from input image.

2. Compute activation map from feature map.

3. Normalize activation map.

4. Average across map within each feature channels.

5. Divide each feature channel by number of maps in that channel.

6. Sum across feature channels.

7. Blur better result.

8. Save descriptive, rescaled

\section{- $\quad$ Feature extraction:}

When we classify the image on the basis of input image needs to extract the features of input image. A feature is defined as capturing a certain visual assets of an image. It is the main task in systems to retrieve the similar images from database similar to query image. In feature extraction, features such as color, texture or shape from image are extracted and creates a feature vector for each image.

$>\quad$ Color:

Color is the most important features that are easily familiar by humans in various images. Color features are the most broadly used in CBIR systems. To take out the color features from an image, a color space and color feature drawing out methods are required. The simplest method to represent colors in an image is to fill color histograms in which a count of the number of pixels of various colors is accumulated. The color base features are as follows.

\section{- HSV histogram}

In HSV (or HSL, or HSB) space is widely used in computer graphics and is a more perceptive way of telling color. Hue, Saturation, Value or HSV is a color representation that describes color (hue or tint) in terms of their shade (saturation or amount of gray) and their brightness (value or luminance). The hue is invariant to the changes in illumination and camera route and hence more suited for object retrieval.

\section{- Auto correlogram}

The color auto correlogram only captures the spatial correlation between the same colors. It integrates the color information and the space information. For each pixel in the image, the auto-correlogram technique needs to go through all the neighbour of that pixel

\section{- Color moment.}

Color moments are used to distinguish images based on their features of color. This moment is used to measure the color match between images. The basis of color moments lies in the statement that the division of color in an image can be interpreted as a probability distribution. If the color in an image follows a certain probability distribution, the moments of that distribution can then be used as features to recognize that image based on color. Three central moments of an images color distribution. They are mean, standard deviation and Skewness. A color can be defined by 3 or more values (Red, Green, and Blue).Moments are planned for each of these channels in an image. The mean, variance and skewnesscolor moments ordered and useful in representing color distributions of images.

\section{$>\quad$ Shape:}

In shape base feature the Gabor wavelet and wallet transform are used.

\section{- $\quad$ Gabor wavelet}

A Gabor wavelet is linear filter used for boundary detection.. Gabor wavelet is expansively adopted to extract texture from the images for retrieval and has been shown to be very capable. Gabor wavelets can be generally measured as orientation- and scale tannable edge and line detectors, and the statistics of these micro features are often used to describe texture information. Basically Gabor filters are a group of wavelets, with each wavelet capturing energy at a exact frequency and correct orientation. The scale and orientation tunable property of Gabor filter makes it mostly useful for texture analysis. Among various wavelet bases, Gabor functions give the best resolution in both the time and frequency domains, and the Gabor wavelet modify seems to be the optimal basis to extract local features for several reasons.

\section{Biological inspiration:}




\section{IARJSET

2. Mathematical and empirical motivation: Gabor wavelet transform has both the multi-resolution and multi-orientation properties and are most constructive for measuring local spatial frequencies. Besidesit has been found to yield deformation tolerance space for pattern detection tasks.

\section{- Wavelet transform}

Wavelet transforms are a mathematical means for performing signal investigation when signal frequency varies over time. For certain classes of signals and images, wavelet analysis provides more accurate information about signal data than other signal study techniques. The wavelet transform uses functions that are limited in both the real and Fourier space.

\section{$>\quad$ SIFT Feature}

Scale Invariant Feature Transform (SIFT) features are features extracted from images to help in loyal matching between different views of the identical object. SIFT is an image local feature clarification algorithm based on scalespace. Due to its well-built matching ability, SIFT has many applications in different fields, such as image retrieval, image stitching, and machine vision. The extracted features are invariant to scale and orientation, and are extremely characteristic of the image. They are extracted in four steps. The first step calculates the locations of potential interest points in the image by detecting the maxima and minima of a set of Difference of Gaussian filters applied at dissimilar scales all over the image. Then, these locations are refined by removal points of low contrast. An orientation is then assigned to each key point based on local image features. Finally, a local feature descriptor is computed at each key point. This descriptor is based on the local image gradient, changed according to the orientation of the key point to provide orientation invariance. Every feature is a vector of dimension 128 uniquely identifying the zone around the key point.

- $\quad$ Classification using SVM Classifier

SVM classifier is a supervised machine learning algorithm which can be used for classification or regression problems. It uses a technique called the kernel trick to change your data and then based on these transformations it finds an optimal boundary between the achievable outputs. In this SVM classifier used to classify images analogous to the input image before re-ranking.

\section{- $\quad$ Re-ranking of images}

For the re-ranking we use spectral clustering and re-ran with click base similarity and typicality. It use image click through data to express image similarity for multiple feature then conduct spectral clustering into group visually and semantically parallel image and finally obtained re-ranking by calculating clicked base cluster typicality and within cluster click based image typicality.This is the first attempt for cluster-based re-ranking using click-through data.We use click-through data and multiple visual modalities simultaneously to learn image similarity, and propose annew similarity learning algorithm, called click based multi-feature similarity learning (CMSL), which conducts metric learning based on click-based triplets selection, while integrating multi-feature into a unified similarity space via multiple kernel learning. We integrate click-through data with image typicality learning to mine the power of this implicit feedback in determining the degree of image relevance to the given query, and further improve the image search performance

\section{EXPERIMENTAL RESULT}

This research performs on re-ranking scheme called spectral clustering re-ranking with clicked based similarity and typicality. In this we give image as well as text query as input. Spectral Clustering form group of images which has similar feature like color, shape, texture. Re-ranking is performed by calculating cluster typicality. From the below diagram we clearly understand the flow of the system.

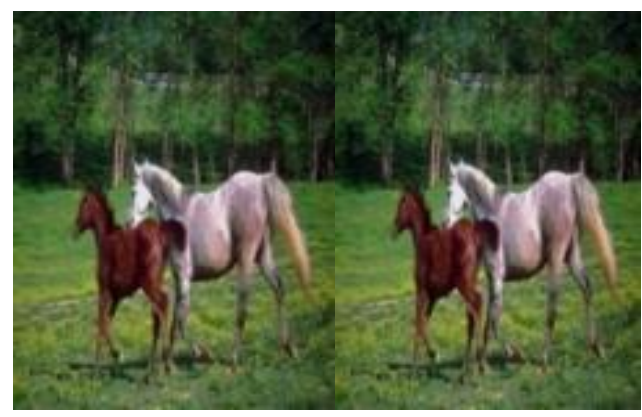

Fig 4.1: input imagefig 4.2: image after preprocessing 
ISO 3297:2007 Certified

Vol. 4, Issue 6, June 2017
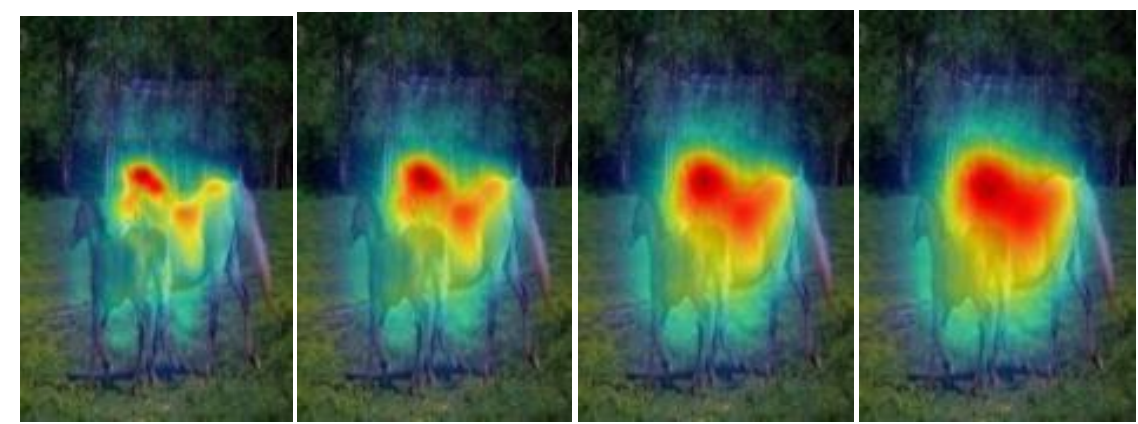

Fig 4.3: saliency map applied at various scale.

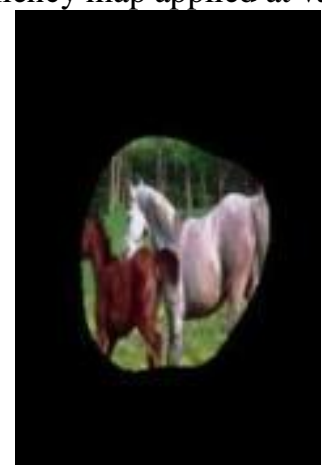

Fig 4.4: rescaled output

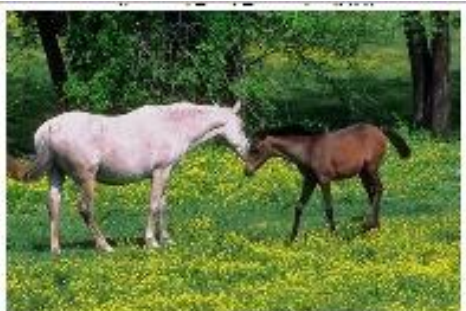

imgros.jpg
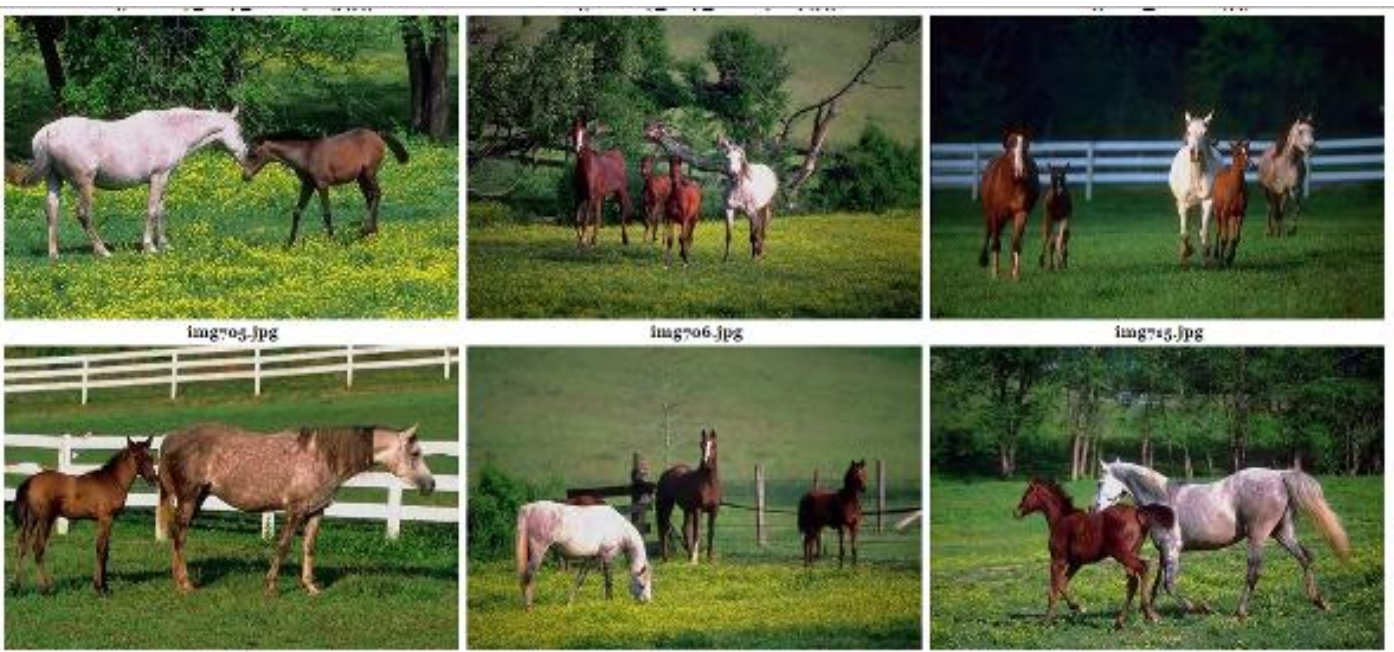

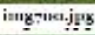

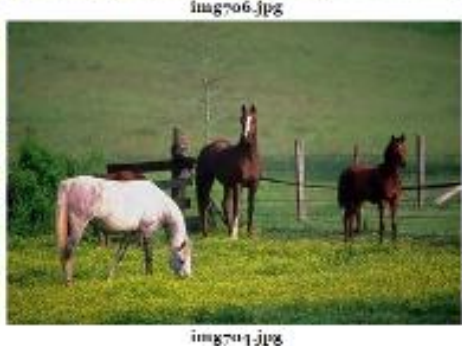

img745.jpg

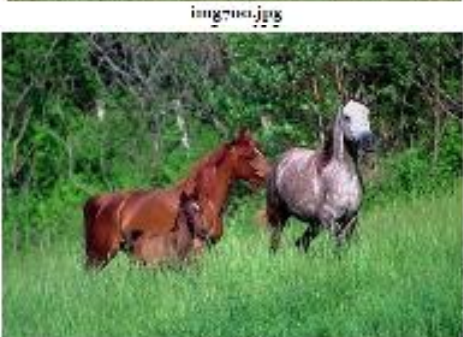

imgon:jpg

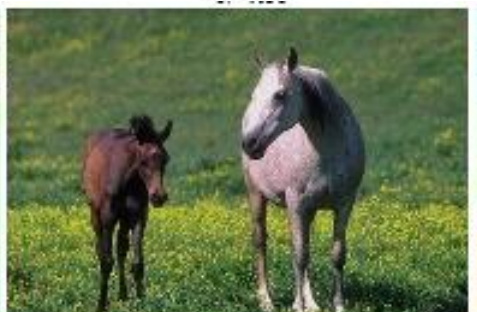

img
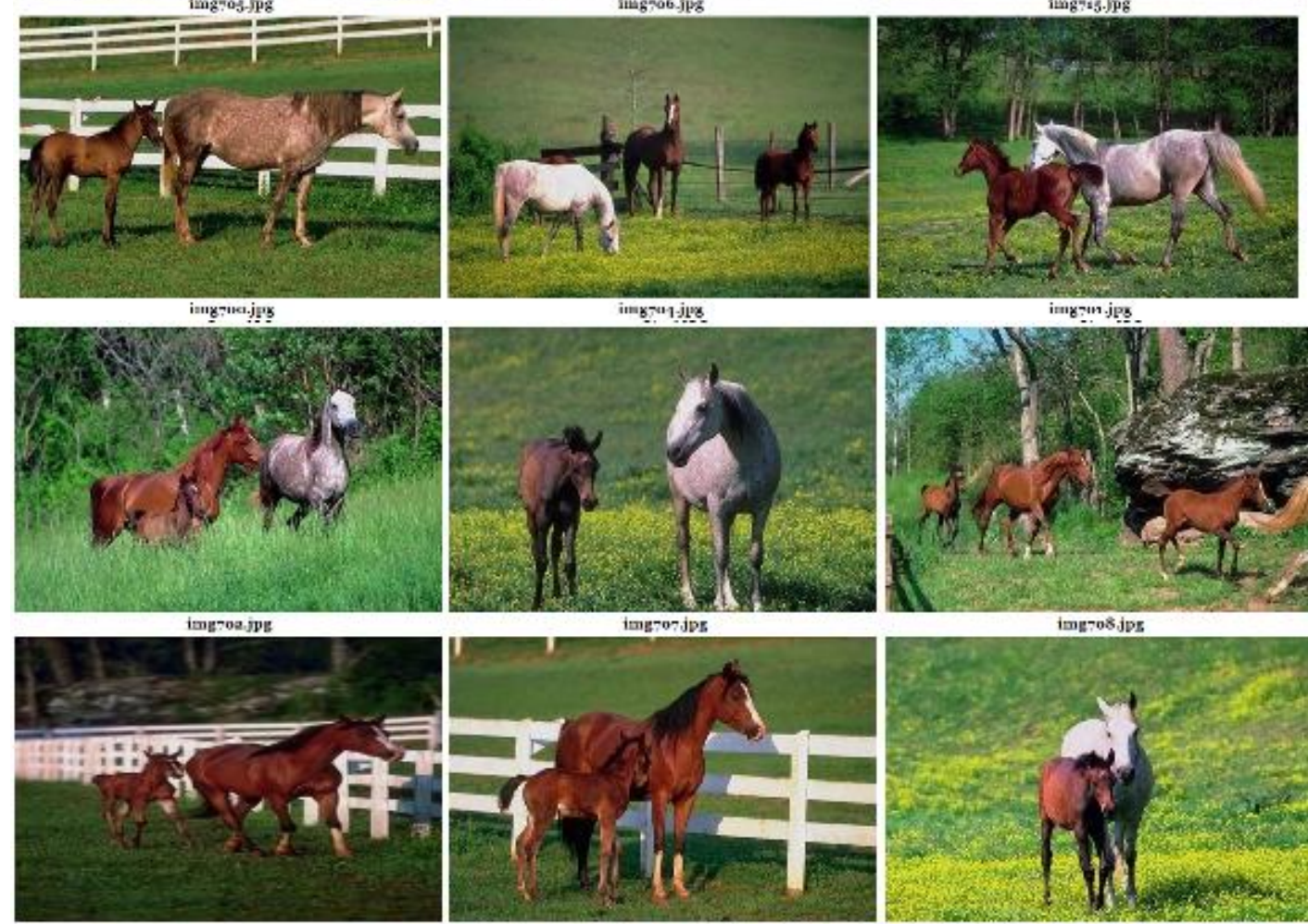

$109740.1 \mathrm{~F}$

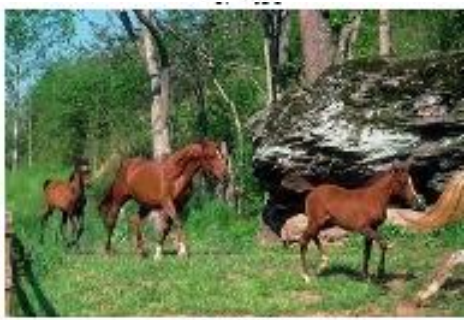

img $70 \$$ jps

1mgrom.1DE

FIG 4.5SEARCH RESULT

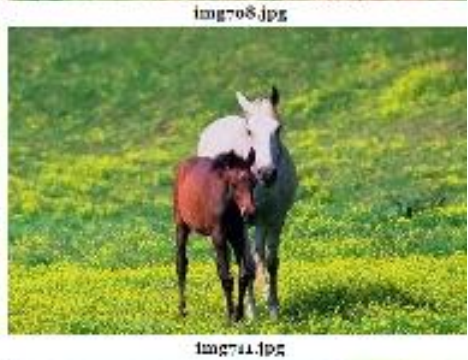

$1 \pm 974.1 \mathrm{DF}$ 
The fig 4.1 is the input image. We can also give the text query as input. Then fig 4.2 is image after preprocessing. Some noise is removed after preprocessing. After that fig 4.3 shows the saliency map of input image at various scale. Saliency map gives highly focused area. The blur is applied in fig 4.3. After that 4.4 shows the rescaled output. This output is use for image searching of similar images. The fig 4.5 shows the similar result images. If the we clicked on one of the images from all result image then these clicked image get first ranked. The fig 4.6 is clicked image and in fig 4.7 the clicked image is in the first position.

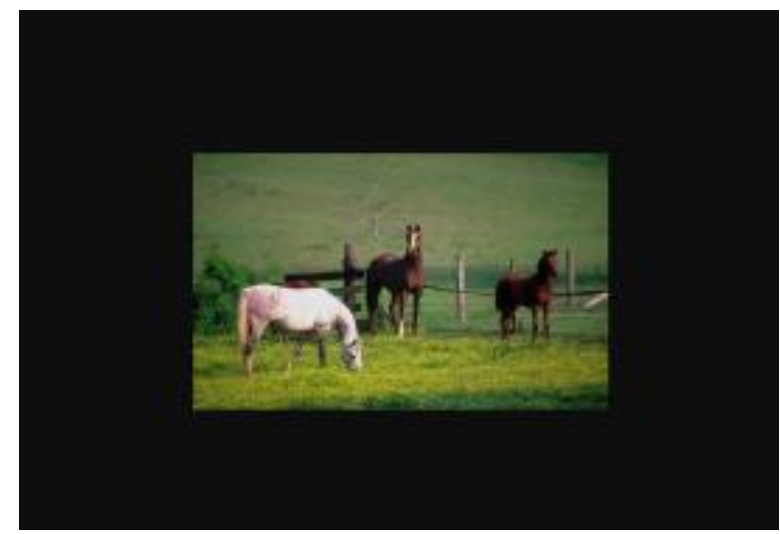

FIG 4.6: CLICKED IMAGE
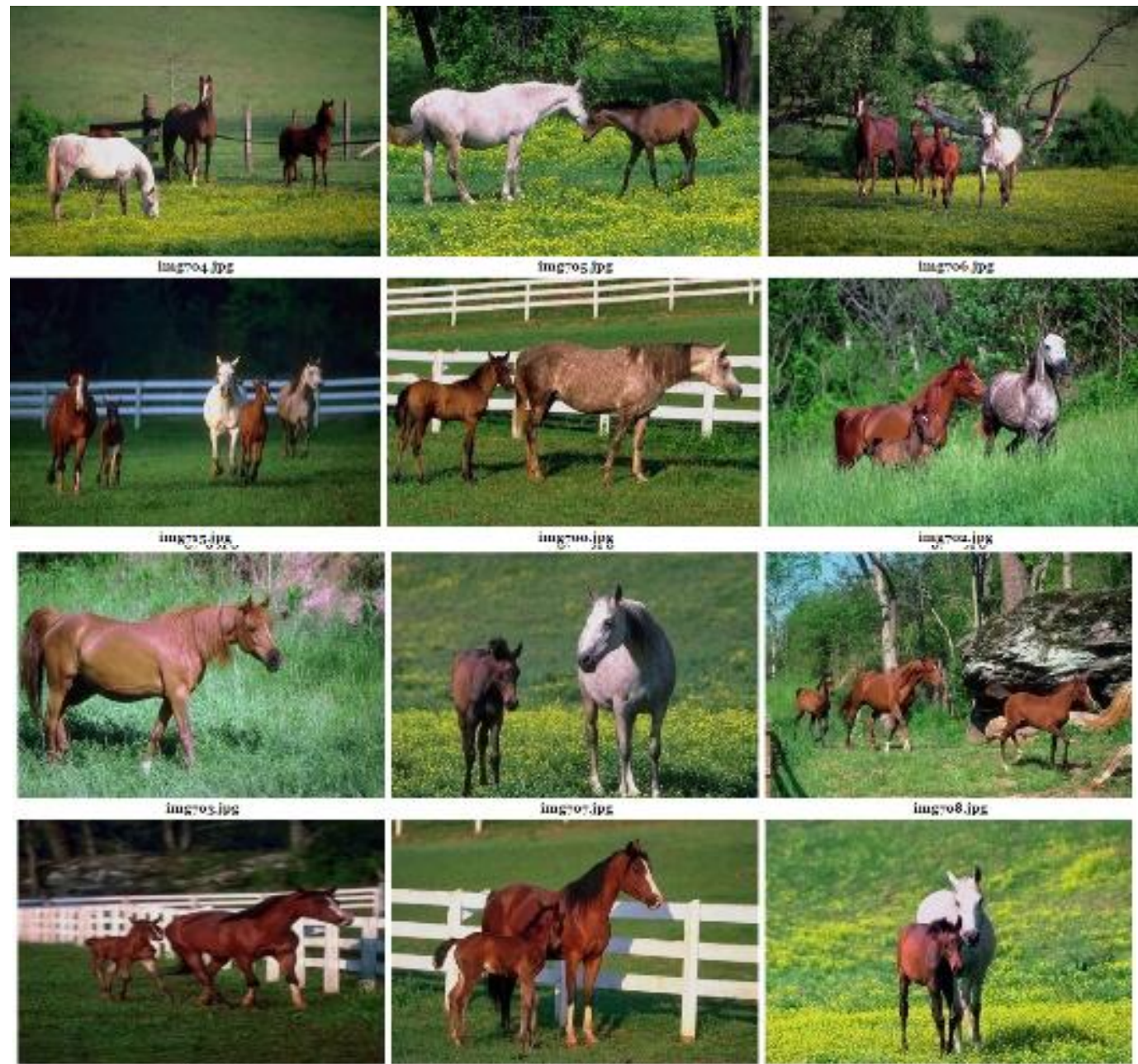

ims $07 . j p 5$

img-o8.jps
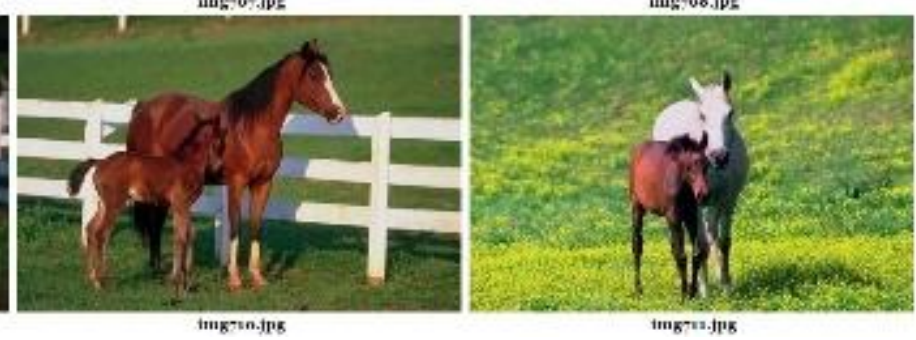

Fig4.7: re-ranking result

In spectral clustering the group of images from which are visually and semantically similar. If we can take example of query like blue lotus car. Then we get the result like in fig 4.8 . 

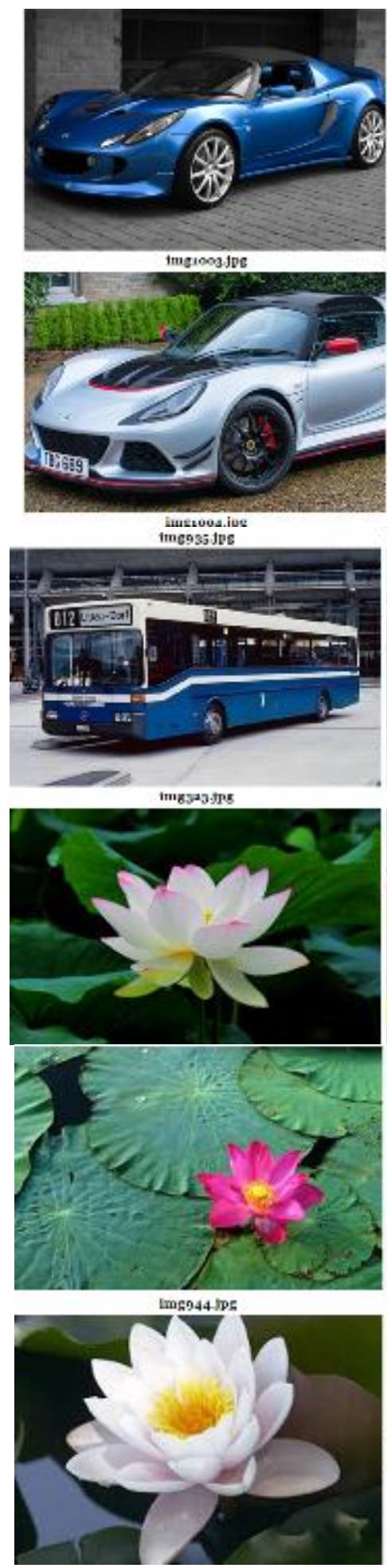
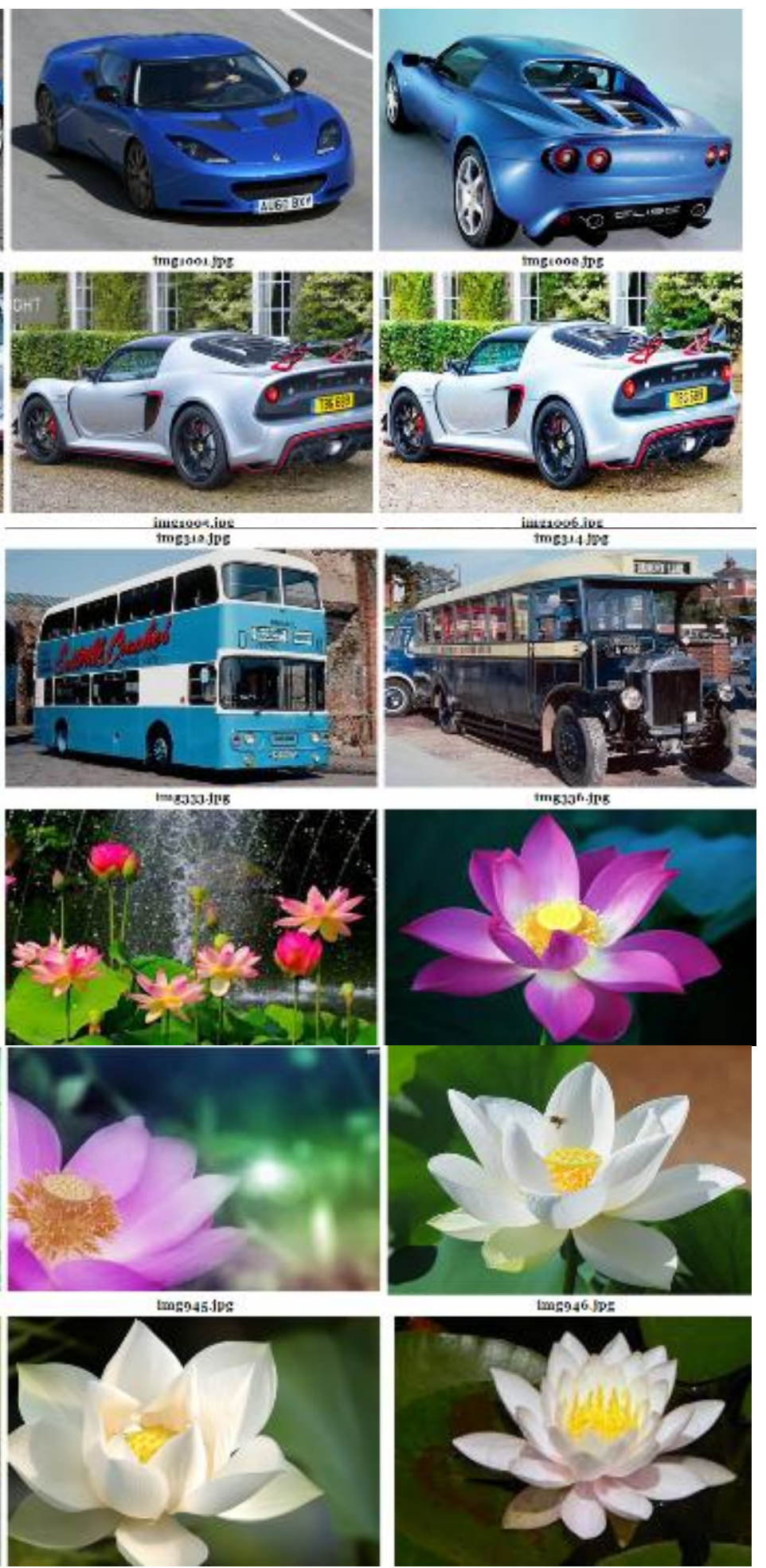

Fig 4.8 spectral clustering

\section{CONCLUSION}

The system is to plan and improve the image search. We can use this system in any type of search engine and in smart phone. The saliencing technique is used to get the better search result in correspondence of the images. After applying saliencing technique we get rescaled output. This output image used as input to get the similar images. The output images are visually and semantically similar. Further we use spectral clustering with clicked base similarity and typicality algorithm. The user gets efficient result as per the requirement. In future can we use neural network to train dataset for more efficient result. 


\section{IARJSET \\ ISSN (Online) 2393-8021 ISSN (Print) 2394-1588 \\ International Advanced Research Journal in Science, Engineering and Technology \\ ISO 3297:2007 Certified \\ Vol. 4, Issue 6, June 2017}

\section{ACKNOWLEDGEMENT}

Initially, we would like to thank our almighty in the success of completing this work. We would expand our gratitude to all the expert for their critical comments.

\section{REFERENCES}

[1] X. Yang, Y. Zhang, T. Yao, C.-W. Ngo, and T. Mei, "Click-boostingmulti-modality graph-based reranking for image search," MultimediaSyst., vol. 21, no. 2, pp. 217-227, 2015.

[2] J. Cai, Z.-J. Zha, M. Wang, S. Zhang, and Q. Tian, "An attribute-assisted reranking model for Web image search,” IEEE Trans. Image Proces

[3] T. Mei, Y. Rui, S. Li, and Q. Tian, "Multimedia search reranking: A literature survey," ACM Comput. Surv., vol. 46, no. 3, p. $38,2014$.

[4] Xiaopeng Yang, Tao Mei, Yongdong Zhang, Jie Liu, and Shin "Web Image Search Re-Ranking With Click-Based Similarity and Typicality" IEEE TRANSACTIONS ON IMAGE PROCESSING, VOL. 25, NO. 10, OCTOBER 2016

[5] B. Wu, T. Mei, W.-H. Cheng, and Y. Zhang, "Unfolding temporal dynamics: Predicting social media popularity using multi-scale temporal decomposition," in Proc. 13th AAAI Conf. Artif. Intell., 2016,pp. 272-278

[6] Y. Zhang, X. Yang, and T. Mei, "Image search re-ranking with query dependentclick-based relevance feedback," IEEE Trans. Image Process., vol. 23, no. 10, pp. 4448-4459, Oct. 2014

[7] Y. Liu, T. Mei, M. Wang, X. Wu, and X.-S.Hua, “Typicality-based visual search reranking,” IEEE Trans. Circuits Syst. Video Technol., vol. 20,no. 5, pp. 749-755, May 2010.

[8] G.-R. Xueet al., "Optimizing Web search using Web click-through data," in Proc. 13th ACM Int. Conf. Inf. Knowl. Manage., 2004, pp. 118126.

[9] Y. Rui, T. S. Huang, M. Ortega, and S. Mehrotra, "Relevance feedback: A power tool for interactive content-based image retrieval," IEEE Trans.Circuits Syst. Video Technol., vol. 8, no. 5, pp. 644-655, Sep. 1998.

[10] X. Tian, D. Tao, X.-S.Hua, and X. Wu, “Active reranking for Web image search,” IEEE Trans. Image Process., vol. 19, no. 3, pp. 805-820, Mar. 2010.

[11] X. S. Zhou and T. S. Huang, "Relevance feedback in image retrieval: A comprehensive review," Multimedia Syst., vol. 8, no. 6, pp. 536-544, Apr. 2003.

[12] V. Jain and M. Varma, "Learning to re-rank: Query-dependent image re-ranking using click data," in Proc. 20th Int. Conf. World Wide Web, 2011, pp. 277-286.

[13] W. Hu, R. Hu, N. Xie, H. Ling, and S. Maybank, "Image classification using multiscale information fusion based on saliency driven nonlinear diffusion filtering," IEEE Trans. Image Process., vol. 23, no. 4, pp. 1513-152

[14] T. Joachims, L. Granka, B. Pan, H. Hembrooke, and G. Gay, "Accuratel interpreting clickthrough data as implicit feedback," in Proc. 28th Annu.Int. ACM SIGIR Conf. Res. Develop. Inf. Retr., 2005, pp. 154-161.

[15] T. Mei, Y. Wang, X.-S. Hua, S. Gong, and S. Li, "Coherent image annotation by learning semantic distance," in Proc. IEEE Conf. Comput.Vis. Pattern Recognit. (CVPR), Jun. 2008, pp. 1-8.6, Apr. 2014

[16] B. Carterette and R. Jones, "Evaluating search engines by modeling the relationship between relevance and clicks," in Proc. Adv. Neural Inf.Process. Syst., 2008, pp. 217-224.

[17] G. Dupret and C. Liao, "A model to estimate intrinsic document relevance from the clickthrough logs of a Web search engine," in Proc.3rd ACM Int. Conf. Web Search Data Mining, 2010, pp. 181-190.

[18] C. Yang, L. Zhang, H. Lu, X. Ruan, and M.-H.Yang, "Saliency detection via graph-based manifold ranking," in Proc. IEEE Conf. Comput. Vis.Pattern Recognit., Jun. 2013, pp. 3166-3173.

[19]J. Harel, C. Koch, and P. Perona, "Graph-based visual saliency," in Proc. Annu. Conf. Neural Inf. Process. Syst., 2007, pp. $545-552$.

[20] G. E. Dupret and B. Piwowarski, "A user browsing model to predict search engine click data from past observations," in Proc. 31st Annu.Int. ACM SIGIR Conf. Res. Develop. Inf. Retr., 2008, pp. 331-338. 